\title{
Rationals, Periodicity and Chaos: A Pythagorean View and a Conjecture into Socio-spatial Dynamics
}

\author{
DIMITRIOS S. DENDRINOS
}

Urban and Transportation Dynamics Laboratory, School of Architecture and Urban Design, The University of Kansas, Lawrence, KS 66045-2250, USA

(Received 30 September 1997; In final form 8 November 1998)

\begin{abstract}
Deep in the fascinating world of numbers there still might lurk useful insights into the processes of the socio-spatial world. A rich section of the world of numbers is of course Number Theory and its pantheon of findings, a part of which is revisited here.

It is suggested in this note that a smooth sequence of seemingly random periodic cycles hides the absence of chaotic dynamics in the sequence. Put differently, a seemingly chaotic sequence of periodic cycles, no matter the bandwidth, implies absence of chaotic motion at any point in the sequence; and conversely, the presence of chaotic motion at any specific point in the sequence implies smooth sequence of periodic cycles at any point in the sequence prior to the onset of quasi periodic or chaotic motions.

To make this conjecture, the paper draws material from the well known property of rational numbers in Number Theory, namely that the division of unity by any integer will always produce a sequence of decimals in some form of periodicity. The conjecture is taken in a liberally interpreted "Pythagorean type" context, whereby a general principle is suggested to be present in all natural or social systems dynamics. Thus, the paper's subtitle.
\end{abstract}

Keywords: Number theory, Rationals, Periodicity, Chaos, Social systems

\section{SOME INTERESTING PERIODIC PROPERTIES INVOLVING THE DECIMALS OF THE UNIT'S CERTAIN FRACTIONS}

In commenting on an initial draft of this paper with its emphasis on computer simulation, Professor M. Sonis has suggested to this author* that the great Swiss mathematician Leonhard Euler was the first one, in the 18th century, to search into the empirical regularities of Theoretical Arithmetics and Number Theory by using numerical calculations. A reemergence of this idea is due to Ulam (1964). Since then, a plethora of papers and books have produced innumerable insights into what it could be perceived as a rather esoteric mathematical topic, enhanced by the modern power of computing. It is suggested here that Number Theory and computing might not be as removed from social sciences as they might first appear.

* Personal correspondence, October 1998. 
A note at the outset: all properties presented below have been obtained by computing; thus, all propositions made here require formal mathematical proofs. No periodicity greater than 75 number cycles is reported, except as a postulate.

What motivated this note, as a part of a series of three closely connected papers, ${ }^{\dagger}$ is the need for a closer look at the approximations involved in various divisions when social stocks are studied. It is not so much the approximation itself which is of import here, but rather the conclusions one might draw from the study into the nature of these divisions proper. More precisely, the paper elaborates on the realization that when shares of stocks are computed then certain properties inevitably appear which characterize these shares. In computing the shares of large stocks (as is the case when urban to regional or to national population ratios are computed), the ensuing probability always consists of a stream of periodic (at times with a very large period) decimals. These periodic sequences are "rationals" as they are the outcome of divisions of integer numbers or fractions, Niven (1961) Chapters 2 and 3.

The study of rationals enjoys a very long and distinguished past in the history of mathematics in general and Number Theory in specific, Adams and Goldstein (1976) Chapter 1. At the start of any rational number sequence should be the study of the unit's divisors by all integers, something that one fails to see in standard textbooks on rationals. Were one to systematically study the behavior of these specific rationals, then one might seek regularities, or distinct properties, governing their periodicity.

As this paper demonstrates, the periodic sequence of decimals of the unit's divisions by integers, although apparently not random, does not seem to obey any predictable rule either; i.e., there does not appear that periodic sequences of increasing period occur at expected intervals as one moves up the magnitude of integer divisors; but neither does it appear that these sequences are random, as one moves up the fractions' scale. Also noteworthy is the fact that small periodic sequences in decimals appear no matter the position in the fractions' scale (from $1 / 1$ to $1 / r$, where $r$ is very large). Since these periodic sequences are to an extent no chance events, the question arises as to whether there are a few underlying principles generating at least certain among them. ${ }^{\ddagger}$ Similar questions arise with divisions involving physical, chemical or other social and economic stocks (for example, intercurrency conversions).

In searching for answers to these initial questions, two general properties underlying periodic cycles in streams of decimals are uncovered: first, there seem to be some prime numbers with associated periodic sequences which dominate; and second, periodic sequences can be constructed as accumulations of successively higher powers of numbers. Both of these properties are uncovered for the first time here, to the author's knowledge.

There seems to be certain linkages between periodic behavior in decimal streams of the unit's divisions, and a variety of events already recorded in numerous mathematical branches, particularly those associated with nonlinear dynamics. Thus, the Pythagorean universality of this paper's title is justified.

\section{A. First Set of General Properties}

A special set of fractions are examined in this paper: that set which represents the unit's division by any other integer. The division produces a stream of decimals which falls into one of the following three distinct categories:

(a) Fixed point This type of decimal stream contains a finite number of digits, at most six decimals in the spectrum $(1 / 2 \ldots 1 / 101)$, involving the division $1 / 64=0.015625$, and at most ten decimals

\footnotetext{
${ }^{\dagger}$ This paper, in conjunction with two other papers by the author titled "Iterates" and "Oddities" (still under construction) comprise the three paper sequence.

${ }^{\ddagger}$ One may ask the question in view of the information we now have on the periodic motions involved in nonlinear dynamics.
} 
in the spectrum $(1 / 2 \ldots 1 / 2001)$ that this paper has looked at (with an aperture of 75 decimal approximation), involving the fraction $1 / 1024=$ 0.0009765625 ; there are fourteen fixed points in the interval $(1 / 2 \ldots 1 / 101)$.

(b) One-number (1-n) cycle This type of decimal sequence usually (but not necessarily) contains two parts: a (varying and nonperiodic) set of decimals (to be called a dendrite, the longest one appearing in the interval $(1 / 2 \ldots 1 / 101)$ at $1 / 96=$ $0.010416 \ldots$, consisting of five digits); and an infinite series of 1's, 2's, 3's, 5's, 6's, 7's or 9's to be referred to as a 1-n.1,1-n.2, . 1-n.9 cycle sequence respectively. The fixed point type decimal sequence could also be looked at as a 1-n.0 cycle with a dendrite. However, its distinct type will be retained, due to the fact that such cycles have an exact (and not an approximate) depiction depicted by its dendrite. There exist two 1-n.1, one 1-n.2, five 1-n.3, one 1-n.5, five 1-n.6, one 1-n.7, and one $1-n .9$ of these cycles (in total 16 out of 101 divisions) in the spectrum $(1 / 2 \ldots 1 / 101)$. Thus, all single digit cycles are encountered with the exception of a 1-n. 4 and a 1-n.8 cycles. The relatively high frequency of 1-n.3 and 1-n.6 periodic cycles is noted in this part of the spectrum. In the spectrum $(1 / 2 \ldots 1 / 2001)$ there are four $1-n .1$, three 1 n.2, fourteen 1-n.3, three 1-n.4, four 1-n.5, thirteen 1-n.6, two 1-n.7, two 1-n.8 and one 1-n.9 period cycles. Again, the relatively high frequency of the $1-n .3$ and 1-n.6 cycles is noted, as well as the relative rarity of the 1-n.9 cycle. Further, it is remarked that as the size of the spectrum increases 20 -fold, the overall frequency of these one-decimal periodic cycles decreases considerably.

(c) A periodic cycle of decimals This type of decimal stream may involve a dendrite. For example, in the interval $1 / 2 \ldots 1 / 101$, at $1 / 88=$ $0.0113636 \ldots$ there is a three digit (011) dendrite and it is followed by a $2-n$ cycle (thirty-six). In the interval $1 / 2 \ldots 1 / 101$ one encounters $2-n$ (seven), 3-n (four), 4-n (one), 5-n (two), 6-n (fifteen), 8-n (one), 9-n (one). In total, there are 31 of these types of periodic cycles in this interval of integer divisors of unity.
The single 4-n periodic cycle is found at the division $1 / 101=0.0099 \ldots$, the single $8-n$ cycle is at $1 / 73=0.01369863 \ldots$, whereas the single $9-n$ periodic cycle corresponds to the division $1 / 81=$ $0.012345679 \ldots$; the relatively high frequency of $3-n$ and particularly 6-n periodic cycles is noted; no 7-n cycle has been encountered in this interval, see

TABLE I Cumulative frequencies of periodic cycles in unit's fractions decimal streams

\begin{tabular}{|c|c|c|c|c|c|c|}
\hline Period & I & II & III & IV & V & VI \\
\hline 2 & 4 & 11 & 24 & 31 & 36 & 40 \\
\hline 3 & 2 & 6 & 19 & 28 & 34 & 39 \\
\hline 4 & - & 1 & 5 & 9 & 12 & 15 \\
\hline 5 & - & 2 & 10 & 18 & 23 & 27 \\
\hline 6 & 9 & 27 & 74 & 109 & 135 & 156 \\
\hline 7 & - & - & 2 & 4 & 7 & 8 \\
\hline 8 & - & 1 & 9 & 17 & 25 & 30 \\
\hline 9 & - & 1 & 3 & 5 & 6 & 7 \\
\hline 10 & - & - & 2 & 3 & 4 & 5 \\
\hline 11 & - & - & - & - & - & - \\
\hline 12 & - & - & 2 & 2 & 4 & 4 \\
\hline 13 & - & 2 & 14 & 24 & 30 & 36 \\
\hline 14 & - & - & - & - & - & - \\
\hline 15 & - & 3 & 13 & 20 & 28 & 33 \\
\hline 16 & 2 & 7 & 20 & 30 & 36 & 44 \\
\hline 17 & - & - & - & - & - & - \\
\hline 18 & 2 & 7 & 25 & 40 & 56 & 67 \\
\hline 19 & - & - & - & - & - & - \\
\hline 20 & - & - & - & - & - & - \\
\hline 21 & - & 2 & 10 & 15 & 20 & 25 \\
\hline 22 & 2 & 6 & 20 & 28 & 37 & 41 \\
\hline 23 & - & - & - & - & - & - \\
\hline 24 & - & - & - & 3 & 4 & 9 \\
\hline 25 & - & $\ldots$ & - & - & - & - \\
\hline 26 & - & - & - & 3 & 4 & 7 \\
\hline 27 & - & - & 2 & 4 & 5 & 6 \\
\hline 28 & - & 3 & 14 & 24 & 32 & 37 \\
\hline 29 & - & - & - & - & - & - \\
\hline 30 & - & - & 9 & 19 & 32 & 42 \\
\hline 31 & - & - & - & - & - & 1 \\
\hline 32 & - & - & 2 & 6 & 12 & 15 \\
\hline 33 & - & 1 & 6 & 9 & 13 & 17 \\
\hline 34 & - & - & 4 & 8 & 12 & 13 \\
\hline 35 & - & 1 & 6 & 11 & 15 & 18 \\
\hline 36 & - & - & - & - & - & 1 \\
\hline 37 & - & - & - & - & - & - \\
\hline 38 & - & - & - & - & - & - \\
\hline 39 & - & - & - & - & 1 & 2 \\
\hline 40 & - & - & - & - & - & - \\
\hline 41 & - & 1 & 5 & 9 & 13 & 15 \\
\hline
\end{tabular}


TABLE I (Continued)

\begin{tabular}{|c|c|c|c|c|c|c|}
\hline Period & I & II & III & IV & $\mathrm{V}$ & VI \\
\hline 42 & - & 2 & 14 & 29 & 42 & 56 \\
\hline 43 & - & - & 2 & 5 & 7 & 9 \\
\hline 44 & - & 1 & 5 & 10 & 14 & 18 \\
\hline 45 & - & - & - & - & - & - \\
\hline 46 & - & 2 & 12 & 21 & 27 & 32 \\
\hline 47 & - & - & - & - & - & - \\
\hline 48 & - & - & 7 & 13 & 20 & 28 \\
\hline 49 & - & - & - & - & - & - \\
\hline 50 & - & - & 1 & 2 & 4 & 6 \\
\hline 51 & - & - & - & 1 & 2 & 3 \\
\hline 52 & - & - & - & 1 & 2 & 3 \\
\hline 53 & - & - & 4 & 7 & 9 & 12 \\
\hline 54 & - & - & - & 1 & 1 & 2 \\
\hline 55 & - & - & - & 1 & 2 & 2 \\
\hline 56 & - & - & - & 1 & 1 & 1 \\
\hline 57 & - & - & - & - & - & - \\
\hline 58 & - & 1 & 7 & 12 & 17 & 19 \\
\hline 59 & - & - & - & 1 & 1 & 1 \\
\hline 60 & - & 1 & 8 & 14 & 19 & 25 \\
\hline 61 & - & - & - & 1 & 2 & 2 \\
\hline 62 & - & - & - & - & - & - \\
\hline 63 & - & - & - & - & - & - \\
\hline 64 & - & - & - & - & - & - \\
\hline 65 & - & - & - & - & - & - \\
\hline 66 & - & - & 5 & 15 & 22 & 32 \\
\hline 67 & - & - & - & - & - & - \\
\hline 68 & - & - & - & 1 & 1 & 1 \\
\hline 69 & - & - & 1 & 3 & 5 & 6 \\
\hline 70 & - & - & - & 1 & 1 & 2 \\
\hline 71 & - & - & - & - & - & - \\
\hline 72 & - & - & - & - & 1 & 2 \\
\hline 73 & - & - & - & - & - & - \\
\hline 74 & - & - & - & - & - & - \\
\hline 75 & - & - & - & - & - & - \\
\hline$>75$ & - & 1 & 102 & 330 & 604 & 908 \\
\hline
\end{tabular}

Note 1: Only periodic motions (plus the dendrite) equal to or less than 75 were examined.

Note 2: The six domains in the spectrum of integer unit divisors, for which cumulative frequencies of periodic sequences were computed, are: I identifies periodic frequencies between 1/2 and $1 / 50$; II identifies periodic frequencies between $1 / 2$ and $1 / 101$; III identifies periodic frequencies between $1 / 2$ and $1 / 500$; IV identifies periodic frequencies between $1 / 2$ and $1 / 1000$; $\mathrm{V}$ identifies periodic frequencies between $1 / 2$ and $1 / 1500$; and VI identifies periodic frequencies between $1 / 2$ and $1 / 2001$.

Table I. In the interval $(1 / 2 \ldots 1 / 2001)$ there are one-hundred and fifty-six 6-n cycles, by far the most frequently encountered periodic sequence in the decimal fractions of unity. Following the 6-n period cycle's frequency, one finds the 18-n (sixty-seven) and 42-n (fifty-six) cycles, Table I. A few hints as to why will be given later.

\section{B. Second Set of General Properties}

In examining the location of the various cycles when they first appear, a number of observations are possible. Period $6-n$ appears at $1 / 7$; the first $16-n$ cycle appears at $1 / 17$; the first $18-n$ at $1 / 19 ; 22-n$ at $1 / 23 ; 28$-n at $1 / 29 ; 46-n$ at $1 / 47 ; 58-n$ at $1 / 59 ; 60$-n at $1 / 61$, Table II. All these periodic sequences (where the period $n$ is at location $1 /(n+1))$, when they first appear, have no dendrite associated with them, Table III, and they correspond to prime number divisors (i.e., $n+1$ are all primes).

The case of the 96-n periodic sequence, appearing at $1 / 97$, is shown in Table III: since 1/97 is not associated with any of the frequently encountered periodic sequences, the hypothesis was tested whether it might be the location of the first 96-n periodic cycle. This proved to be the case. Consequently, one might expect that prime number divisors, designated as $N$, are either points in the spectrum of numbers associated with a set of frequently encountered periodic sequences, or the beginning point of $N-1$ periodic cycles.

Period $15-\mathrm{n}$ appears at $1 / 31 ; 21-\mathrm{n}$ at $1 / 43 ; 33-\mathrm{n}$ at $1 / 67 ; 35-n$ at $1 / 71 ; 41-n$ at $1 / 83 ; 44-n$ at $1 / 89 ; 53-n$ at $1 / 107$, Table II. All these periodic sequences (where the period $n$ is at location $1 /(2 n+1))$, when they first appear have no dendrite associated with them, too, and they also correspond to prime number divisors (i.e., $2 n+1$ are all primes), Table III.

When the 43-n period sequence first appears at $1 / 173$, Table II, it happens that $173=4 n+1 ; 69-\mathrm{n}$ at $1 / 277$, where $277=4 n+1$, Table III.

The 34-n period first appears at $1 / 103$, where $103=3 n+1$, Table III. 103 is also a prime number.

The sequence in obtaining periodic streams of decimals as the magnitude of the divisors increase, Table III, observes the following rules: first, a fixed point is obtained at $1 / 2=0.5$; it is followed by a $1-n .3$ cycle at $1 / 3=0.3 \ldots$; at $1 / 7=0.142857 \ldots$ the first six period $(6-n)$ cycle appears; at $1 / 11=0.09 \ldots$ 
TABLE II First appearance of periodic sequences

\begin{tabular}{|c|c|c|c|c|c|c|c|c|c|}
\hline Period & $\begin{array}{c}\text { First } \\
\text { appearing at }\end{array}$ & Period & $\begin{array}{c}\text { First } \\
\text { appearing at }\end{array}$ & Period & $\begin{array}{c}\text { First } \\
\text { appearing at }\end{array}$ & Period & $\begin{array}{c}\text { First } \\
\text { appearing at }\end{array}$ & Period & $\begin{array}{c}\text { First } \\
\text { appearing at }\end{array}$ \\
\hline 2 & 11 & 17 & $?$ & 32 & 353 & 47 & $?$ & 62 & $?$ \\
\hline 3 & 27 & 18 & 19 & 33 & 67 & 48 & 119 & 63 & $?$ \\
\hline 4 & 101 & 19 & $?$ & 34 & 103 & 49 & $?$ & 64 & $?$ \\
\hline 5 & 41 & 20 & $?$ & 35 & 71 & 50 & 251 & 65 & $?$ \\
\hline 6 & 7 & 21 & 43 & 36 & 1919 & 51 & 613 & 66 & 161 \\
\hline 7 & 239 & 22 & 23 & 37 & $?$ & 52 & 521 & 67 & $?$ \\
\hline 8 & 73 & 23 & $?$ & 38 & $?$ & 53 & 107 & 68 & 920 \\
\hline 9 & 81 & 24 & 511 & 39 & 1431 & 54 & 856 & 69 & 277 \\
\hline 10 & 324 & 25 & $?$ & 40 & $?$ & 55 & 760 & 70 & 781 \\
\hline 11 & $?$ & 26 & 583 & 41 & 83 & 56 & 928 & 71 & $?$ \\
\hline 12 & 390 & 27 & 243 & 42 & 49 & 57 & $?$ & 72 & 1387 \\
\hline 13 & 53 & 28 & 29 & 43 & 173 & 58 & 59 & 73 & $?$ \\
\hline 14 & $?$ & 29 & $?$ & 44 & 89 & 59 & 610 & 74 & $?$ \\
\hline 15 & 31 & 30 & 211 & 45 & $?$ & 60 & 61 & 75 & $?$ \\
\hline 16 & 17 & 31 & 1621 & 46 & 47 & 61 & 733 & & \\
\hline
\end{tabular}

the first 2-n period cycle is encountered; at $1 / 17$ the first 16-n periodic sequence is obtained, whereas at 1/19 the first 18-n cycle comes up.

The division $1 / 81=0.0123456790 \ldots$ produces the first and only $9-n$ period cycle in the interval $(1 / 2 \ldots 1 / 101)$, whereas, the division $1 / 101=$ $0.0099 \ldots$ results in the first and only 4-n cycle in the set of divisions within the above interval (interval II of Table II).

With the exception of the first fixed point (at $1 / 2$ ) and the case of the first $3-n$ period cycle (at $1 / 27$ ), all other transitions to a new phase involve divisions by a prime number.

A 3-n periodic cycle commences, as already noted, at $1 / 27$ (where 27 is not a prime number); it appears next at $1 / 37$, where 37 is a prime number. One may ask why is it so, and what particular value is associated with $27=3^{3}$. This topic will be addressed at another occasion. Note that $1 / 27=$ $0.037037 \ldots$ and $1 / 37=0.027027 \ldots$

In general, periodicity associated with prime number divisors has always zero length dendrites. Put differently, if there is a dendrite in the periodic sequence of decimals in a unit's divisor, then this divisor is not a prime number; but the opposite does not necessarily hold, since periodic sequencing without dendrites might involve nonprime number divisors.

\section{DOMINANCE OF CERTAIN PRIME NUMBER DIVISORS}

When unity is divided by a multiple of certain prime numbers, then some dominance patterns emerge. Some of these dominance patterns are as follows:

Rule 1 The divisor $11 \times 5=55$ will behave as the divisor of 11 (i.e., it will exhibit a 2-n periodic cycle). Thus, prime number 11 dominates prime number 5 , or $11 D 5$.

Rule 2 The divisor $7 \times 11=77$ will behave as the divisor of 7 (i.e., it will exhibit a 6 -n periodic cycle). Thus, prime number 7 dominates prime number 11, or $7 D 11$.

Rule 3 Since $7 D 11$ and $11 D 5$ it must follow that $7 D 5$ which is the case. For example, $1 / 35$ behaves as $1 / 7$ (i.e., it exhibits a 6 - $n$ periodic cycle).

Rule 4 The divisor $3 \times 5=15$ will behave as the divisor 3, thus 3D5.

Rule 5 The divisor $7 \times 3=21$ will behave as the divisor 7, thus $7 D 3$.

Rule 6 The divisor $3 \times 11=33$ will behave as the divisor 11, thus $11 D 3$.

Comment Consequently, so far, the following dominance pattern has been established: 7D11D3D5. 
TABLE III The Periodic Table of unit fractions by integers less than 103*

\begin{tabular}{|c|c|c|c|}
\hline Division & Behavior & Comment & $\begin{array}{c}\text { Prime } \\
\text { divisor }(p)\end{array}$ \\
\hline $1 / 2=0.5$ & fixed point (fp) & & \\
\hline $1 / 3=0.333 \ldots$ & $1-n .3$ cycle & first $1-n$ cycle & $\mathrm{p}$ \\
\hline $1 / 4=0.25$ & $\mathrm{fp}$ & & \\
\hline $1 / 5=0.2$ & $\mathrm{fp}$ & & $\mathrm{p}$ \\
\hline $1 / 6=0.16 \ldots$ & $1-n .6$ cycle & one digit dendrite & \\
\hline $1 / 7=0.142857 \ldots$ & 6-n cycle & first 6 -n cycle & $\mathrm{p}$ \\
\hline $1 / 8=0.125$ & $\mathrm{fp}$ & & \\
\hline $1 / 9=0.111 \ldots$ & 1-n.1 cycle & & \\
\hline $1 / 10=0.1$ & $\mathrm{fp}$ & & \\
\hline $1 / 11=0.09 \ldots$ & 2-n cycle & first $2-n$ cycle & $\mathrm{p}$ \\
\hline $1 / 12=0.083 \ldots$ & $1-\mathrm{n} .3$ cycle & two digit dendrite & \\
\hline $1 / 13=0.076923 \ldots$ & 6-n cycle & & $\mathrm{p}$ \\
\hline $1 / 14=0.0714285 \ldots$ & 6-n cycle & one digit dendrite & \\
\hline $1 / 15=0.06 \ldots$ & $1-$ n.6 cycle & one digit dendrite & \\
\hline $1 / 16=0.0625$ & $\mathrm{fp}$ & & \\
\hline $1 / 17=0.0588235294117647 \ldots$ & $16-\mathrm{n}$ cycle & first $16-n$ cycle & $\mathrm{p}$ \\
\hline $1 / 18=0.05 \ldots$ & $1-\mathrm{n} .5$ cycle & one digit dendrite & \\
\hline $1 / 19=0.052631578947368421 \ldots$ & 18-n cycle & & $\mathrm{p}$ \\
\hline $1 / 20=0.05$ & $\mathrm{fp}$ & & \\
\hline $1 / 21=0.0476190 \ldots$ & 6-n cycle & & \\
\hline $1 / 22=0.045 \ldots$ & 2-n cycle & one digit dendrite & \\
\hline $1 / 23=0.0434782695652173913 \ldots$ & 22-n cycle & & $\mathrm{p}$ \\
\hline $1 / 24=0.0416 \ldots$ & 1-n.6 cycle & three digit dendrite & \\
\hline $1 / 25=0.04$ & $\mathrm{fp}$ & & \\
\hline $1 / 26=0.0384615 \ldots$ & 6-n cycle & one digit dendrite & \\
\hline $1 / 27=0.037 \ldots$ & $3-n$ cycle & first $3-n$ cycle & \\
\hline $1 / 28=0.0357142857 \ldots$ & 6-n cycle & four digit dendrite & \\
\hline $1 / 29=0.0344827586206896551724137931 \ldots$ & 28-n cycle & & $\mathrm{p}$ \\
\hline $1 / 30=0.03 \ldots$ & $1-$ n.3 cycle & one digit dendrite & \\
\hline $1 / 31=0.032258064516129 \ldots$ & $15-\mathrm{n}$ cycle & & $\mathrm{p}$ \\
\hline $1 / 32=0.03125$ & $\mathrm{fp}$ & & \\
\hline $1 / 33=0.030 \ldots$ & 2-n cycle & & \\
\hline $1 / 34=0.02941176470588235 \ldots$ & 16-n cycle & one digit dendrite & \\
\hline $1 / 35=0.0285714 \ldots$ & 6-n cycle & one digit dendrite & \\
\hline $1 / 36=0.027 \ldots$ & 1-n.7 cycle & two digit dendrite & \\
\hline $1 / 37=0.027 \ldots$ & 3-n cycle & & $\mathrm{p}$ \\
\hline $1 / 38=0.0263157894736842105 \ldots$ & 18-n cycle & one digit dendrite & \\
\hline $1 / 39=0.025641 \ldots$ & 6-n cycle & & \\
\hline $1 / 40=0.025$ & $\mathrm{fp}$ & & \\
\hline $1 / 41=0.02439 \ldots$ & $5-n$ cycle & first 5-n cycle & $\mathrm{p}$ \\
\hline $1 / 42=0.0238095 \ldots$ & 6-n cycle & one digit dendrite & \\
\hline $1 / 43=0.023255813953488372093 \ldots$ & 21-n cycle & & $\mathrm{p}$ \\
\hline $1 / 44=0.0227 \ldots$ & 2 -n cycle & two digit dendrite & \\
\hline $1 / 45=0.0222 \ldots$ & $1-$ n. 2 cycle & one digit dendrite & \\
\hline $1 / 46=0.02173913043478260869565 \ldots$ & $22-n$ cycle & one digit dendrite & \\
\hline $1 / 47=0.0212765957446808510638297872340425531914893617 \ldots$ & 46-n cycle & & $\mathrm{p}$ \\
\hline $1 / 48=0.02083 \ldots$ & 1-n.3 cycle & four digit dendrite & \\
\hline $1 / 49=0.020408163265306122448979591836734693877551 \ldots$ & 42-n cycle & & \\
\hline $1 / 50=0.02$ & $\mathrm{fp}$ & & \\
\hline $1 / 51=0.01960784313725490 \ldots$ & $16-n$ cycle & & \\
\hline $1 / 52=0.01923076 \ldots$ & 6-n cycle & two digit dendrite & \\
\hline $1 / 53=0.0188679245283 \ldots$ & 13 -n cycle & first $13-n$ cycle & $\mathrm{p}$ \\
\hline $1 / 54=0.0185 \ldots$ & 3 -n cycle & one digit dendrite & \\
\hline
\end{tabular}


TABLE III (Continued)

\begin{tabular}{|c|c|c|c|}
\hline Division & Behavior & Comment & $\begin{array}{c}\text { Prime } \\
\text { divisor }(p)\end{array}$ \\
\hline $1 / 55=0.018 \ldots$ & 2-n cycle & one digit dendrite & \\
\hline $1 / 56=0.017857142$. & 6-n cycle & three digit dendrite & \\
\hline $1 / 57=0.017543859649122807 \ldots$ & 18-n cycle & & \\
\hline $1 / 58=0.01724137931034482758620689655 \ldots$ & 28-n cycle & one digit dendrite & \\
\hline $1 / 59=0.0169491525423728813559322033898305084745762711864406779661 \ldots$ & 58-n cycle & & $\mathrm{p}$ \\
\hline $1 / 60=0.016 \ldots$ & $1-n .6$ cycle & two digit dendrite & \\
\hline $1 / 61=0.016393442622950819672131147540983606557377049180327868852459 \ldots$ & $60-\mathrm{n}$ cycle & & $\mathrm{p}$ \\
\hline $1 / 62=0.0161290322580645 \ldots$ & 15-n cycle & one digit dendrite & \\
\hline $1 / 63=0.015873 \ldots$ & 6-n cycle & & \\
\hline $1 / 64=0.015625$ & $\mathrm{fp}$ & & \\
\hline $1 / 65=0.0153846 \ldots$ & 6-n cycle & one digit dendrite & \\
\hline $1 / 66=0.015 \ldots$ & 2-n cycle & one digit dendrite & \\
\hline $1 / 67=0.0149253731343283582088955223880597 \ldots$ & 33-n cycle & & $\mathrm{p}$ \\
\hline $1 / 68=0.014705882352941176$. & 16-n cycle & & \\
\hline $1 / 69=0.0144927536231884057971 \ldots$ & 22-n cycle & & \\
\hline $1 / 70=0.0142857 \ldots$ & 6-n cycle & one digit dendrite & \\
\hline $1 / 71=0.01408450704225352112676056338028169$. & 35-n cycle & & $\mathrm{p}$ \\
\hline $1 / 72=0.0138 \ldots$ & 1-n.8 cycle & three digit dendrite & \\
\hline $1 / 73=0.01369863 \ldots$ & 8-n cycle & first 8-n cycle & $\mathrm{p}$ \\
\hline $1 / 74=0.0135 \ldots$ & 3-n cycle & one digit dendrite & \\
\hline $1 / 75=0.013 \ldots$ & $1-n .3$ cycle & two digit dendrite & \\
\hline $1 / 76=0.01315789473684210526 \ldots$ & 18-n cycle & two digit dendrite & \\
\hline $1 / 77=0.012987 \ldots$ & 6-n cycle & & \\
\hline $1 / 78=0.0128205 \ldots$ & 6-n cycle & one digit dendrite & \\
\hline $1 / 79=0.0126582278481 \ldots$ & 13-n cycle & & $\mathrm{p}$ \\
\hline $1 / 80=0.0125$ & $\mathrm{fp}$ & & \\
\hline $1 / 81=0.012345679 \ldots$ & 9-n cycle & first 9-n cycle & \\
\hline $1 / 82=0.012195 \ldots$ & 5-n cycle & one digit dendrite & \\
\hline $1 / 83=0.01204819277108433734939759036144578313253$. & 41-n cycle & & $\mathrm{p}$ \\
\hline $1 / 84=0.01190476 \ldots$ & 6-n cycle & two digit dendrite & \\
\hline $1 / 85=0.01176470588235294 \ldots$ & 16-n cycle & one digit dendrite & \\
\hline $1 / 86=0.0116279069767441860465 \ldots$ & 21-n cycle & one digit dendrite & \\
\hline $1 / 87=0.0114942528735632183908045977 \ldots$ & 28-n cycle & & \\
\hline $1 / 88=0.01136 \ldots$ & 2-n cycle & three digit dendrite & \\
\hline $1 / 89=0.01123595505617977528089887640449438202247191 \ldots$ & 44-n cycle & & $\mathrm{p}$ \\
\hline $1 / 90=0.01 \ldots$ & $1-n .1$ cycle & one digit dendrite & \\
\hline $1 / 91=0.010989 \ldots$ & 6-n cycle & & $\mathrm{p}$ \\
\hline $1 / 92=0.010869565217391304347826 \ldots$ & 22-n cycle & two digit dendrite & \\
\hline $1 / 93=0.010752688172043 \ldots$ & 15-n cycle & & \\
\hline $1 / 94=0.01063829787234042553191489361702127659574468085 \ldots$ & 46-n cycle & one digit dendrite & \\
\hline $1 / 95=0.0105263157894736842 \ldots$ & 18-n cycle & one digit dendrite & \\
\hline $1 / 96=0.010416 \ldots$ & $1-$ n. 6 cycle & five digit dendrite & \\
\hline $1 / 97=0.0103092 \ldots$ & $96-\mathrm{n}$ cycle** & & $\mathrm{p}$ \\
\hline $1 / 98=0.010204816326530612244897959183673469387755 \ldots$ & 42-n cycle & one digit dendrite & \\
\hline $1 / 99=0.01 \ldots$ & 2-n cycle & & \\
\hline $1 / 100=0.01$ & $\mathrm{fp}$ & & \\
\hline $1 / 101=0.0099 \ldots$ & 4-n cycle & first 4-n cycle & $\mathrm{p}$ \\
\hline $1 / 102=0.00980392156862745 \ldots$ & 16-n cycle & one digit dendrite & \\
\hline
\end{tabular}

*All calculations were carried out at a 75 -digit approximation.

**As an experiment, the $1 / 97$ sequence was computed to test whether a prior "expected" 96 -n cycle would indeed appear, by using 120 decimals in length sequence; the expectation was realized. See text for more discussion. 
Rule 7 The divisor $13 \times 11=143$ will exhibit the behavior of 13 , i.e., a $6-n$ period cycle. Thus: 13D11D3D5. Among primes 7 and 13 there does not arise a question of dominance, since both produce 6-n period cycles.

Rule 8 The divisor of unity $17 \times 7=119$ (or $17 \times 13=221)$ will behave neither as that of 17 (with a 16-n cycle) nor that of 7 and 13 (with a 6-n cycle); in effect $1 / 119$ and $1 / 221$ both exhibit a 48 $\mathrm{n}$ cycle. Thus, the $16-\mathrm{n}$ cycle resulting from $1 / 17$ is a killer prime of frequencies with respect to both 7 and 13. Consequently, here lies the answer to the initial question motivating this paper: large number divisors of the unit tend invariably to produce very large number periodic streams because of the killer properties of the 16-n cycle over all other smaller period streams.

Comment The killer property of 17 (and 19) and the relative dominance of 7 and 13 among prime numbers may hint at the high frequency of the 16$\mathrm{n}$ (and the 18-n), as well as that of the 6-n period cycles in the Periodic Table (Table I). Thus, 17 may be dominant not because there are many multiples of 17 in any interval (I, II, .., VI), but because of its killer property.

The same rationale may apply to 7 and 13 with regards to the 6-n cycle, as well. Consequently, dominance is afforded a prime number because of the frequency of its underlying behavior, and not because of its relative magnitude.

Rule 10 Any divisor of unity by an integer which is not a prime but a multiple of one or more prime(s) will behave as the dominant prime number. All prime numbers dominate all nonprime numbers which are not multiples of primes.

Professor Sonis" has remarked to the author, in response to a prior draft of this paper, that the dual representation of the rational numbers will result in the symbolic dynamics introduced by Thue (1906) and Morse (1921), and used by Metropolis et al. (1973) for the presentation of "universal sequences," see Schroeder (1991) and Hao (1983).
One might profitably ponder the question what the dominance patterns, identified above, and the cycle accumulation phenomenon (to be addressed below), might imply for these universal sequences. Among other things, they might set "markers" for separation domains in these sequences.

\section{ON THE NATURE OF CERTAIN PERIODIC BEHAVIOR: CYCLE ACCUMULATION}

The discussion which follows, beyond its mathematical interest, presents the opportunity to derive some fundamental underlying principles in sociospatial (and possibly even natural) systems. It is shown that the decimal sequences of certain rational numbers is but an accumulation of a well identified sequence of cycles. This finding may shed some light into the nature of socio-spatial periodic dynamics, as the composites of temporal (hourly, daily, weekly, monthly, seasonal, yearly, etc.) cycles; as well as spatially dominant (urban, regional, national, global) cycles.

First, a brief look into some periodic decimal streams will be taken. The inverse of 7 is a $6-n$ cycle $(1 / 7=0.142857 \ldots)$; this cycle is simply the accumulation of successively higher powers of 2 , multiplied by 7 , and occupying two decimal places each, as follows:

$\begin{array}{rrrrrrrr}14 & 28 & 57 & 14 & 28 & 57 \ldots & & \\ & & 56 & & & & & \\ & & 1 & 12 & & & & \\ & & & 2 & 24 & & & \\ & & & & 4 & 48 & & \\ & & & & & 8 & 96 & \\ - & - & - & - & - & - & - & - \\ 14 & 28 & 57 & 14 & 28 & 56 & & \\ & & & & & 1 & 13 & 92 \\ & & & & - & - & - \\ & & & & 57 & \ldots & \ldots\end{array}$

I Private correspondence, October 1998. 
so that: $14=2 \times 7 ; 28=2^{2} \times 7 ; 56=2^{3} \times 7 ; 112=$ $2^{4} \times 7 ; \quad 224=2^{5} \times 7 ; \quad 448=2^{6} \times 7 ; \quad 896=2^{7} \times 7 ;$ $1792=2^{8} \times 7$, etc. The accumulation of these powers of 2 occurs as indicated above.

Another similar cycle is the inverse of 13 , another 6-n cycle: $0.076923 \ldots$ This specific cycle involves again two decimal spaces and successively higher powers of 9 multipied by 7 as follows:

0.07 63

$\begin{array}{ccccc} & 5 & 67 & & \\ - & & 51 & 03 & \ldots \\ 0.07 & - & - & - & \\ & 68 & & & \\ & 1 & 18 & 03 & \ldots \\ & - & - & - & \end{array}$

so that, $7=7 \times 9^{0} ; 63=7 \times 9^{1} ; 567=7 \times 9^{2} ; 5103=$ $7 \times 9^{3}$, etc ... The accumulation occurs as indicated above with decimal units carried over to the appropriate locations and with the additions shown under the lines drawn.

Next, a longer periodic stream of decimals is analyzed. Consider the inverse of 19 which is a 18-n cycle, see Table III. This is a two-decimal spaces accumulation of simple powers of 5 , as shown below:

$\begin{array}{rrrrrrr}0.05 & 25 & & & & & \\ & 1 & 25 & & & & \\ & & 6 & 25 & & & \\ & & & 31 & 25 & & \\ & & & 1 & 56 & 25 & \ldots \\ - & - & - & - & - & - & \\ 0.05 & 26 & 31 & 57 & 81 & \ldots & \end{array}$

so that the terms shown above involve the first (5), second (25), third (125), fourth (625), fifth (3125) and sixth (15625) powers of 5 . They have been accumulated appropriately. Thus, the $18-\mathrm{n}$ periodic stream of decimals is nothing but a sequence and accumulation of simple powers of 5 . The key is that each of these successively higher powers of 5 occupy two decimal spaces.
Some definitions and nomenclature: In the following 6-n sequence (or power accumulation) involving the inverse of 112 (an integer equal to $\left.2^{4} \times 7\right)$ and a four digit dendrite (0089), Table III, $1 / 112=0.0089285714285714 \ldots$ one observes the following: there is a two-digit space over which products of 91 accumulate. The two-decimal spaces area will be referred to as the box- 2 space; the first 0 which is outside the boxed area is of course the one digit dendrite of the power accumulation sequence; the prime number 7 (seemingly a prime number is always present in a periodic sequence broken down to an accumulation of products or powers of usually another number) is the base; the number 91 , products of which here accumulate $(91,182,364,728$, etc.), is a product seed.

If powers of the seed accumulate, like in the case of $1 / 19$, the example previously shown, then this is a power seed; the product number (here 2 , as the seed is successively doubled) is the multiplier; in the case of exponentiation, it would be referred to as the power.

Another solved case of a periodic stream involving power accumulation is that of $1 / 47=$ $0.02127659574 \ldots$ a $46-n$ periodic sequence, see Table III. This sequence involves an accumulation of powers of 6 (the power seed $6=2 \times 3$, prime number 3 being the base), with 02 being the twodigit power accumulation dendrite; the box- 2 space allows for accumulation of: $2 \times 6^{2}=72,2 \times 6^{3}=$ $432,2 \times 6^{4}=2592,2 \times 6^{5}=15552$.

An informative behavior, see the paper on "Oddities" by the author, regarding rules involved in series of sequences, is found in the periodic streams of the region of inverses for the numbers 991-999: the 1/998 (a periodic sequence greater than 75 digits) is formed by accumulation of powers of 2 over a box-4 space; 1/997 (also a periodic sequence with a period greater than 75 digits) involves powers of $3 ; 1 / 996$ (a 41-n periodic sequence) is an accumulation of powers of $4 ; 1 / 995$ (a periodic sequence with period greater than 75) involves powers of $5 ; 1 / 994$ (with a period greater than 75 , too) is an accumulation of powers of $6 ; 1 / 993$ (with a period greater than 75 , as well) involves powers 
of 7; 1/992 (a 15-n periodic cycle) has powers of 8 ; 1/991 (with a period greater than 75) has powers of 9; and 1/990 (a 2-n periodic cycle) involves powers of 10. A similar case is found in the region of inverses at 490-499.

But there are other periodic streams of decimals which do not succumb to such power accumulation; an informative example is the inverse of the first 16-n cycle stream encountered in the Periodic Table, that of 17. This finding forces one to recognize the existence of at least two types of periodic streams, a false (like the one of 1/19) and a genuine one (like that of $1 / 17$ ).

\section{OTHER SOLVED SEQUENCES OF POTENTIAL INTEREST}

Next, a number of other sequences are presented, because they seem to indicate some fertile grounds for further work. All of the sequences given below have been solved in terms of their underlying principles giving rise to them. Since these cases identify sequences consisting of variations of previously established themes, they might possibly be indicating that the study of rationals hides still yet more interesting cases.

Take for instance the inverse of 970: $1 / 970=$ $0.00103092783505 \ldots$; this is a periodic sequence with a period greater than 75 digits, with products of 10 accumulating over two-space areas. The exact specifications are: box-2, two-digit power accumulation dendrite $(00)$, prime number 3 is the base, number 10 , is the product seed, number 3 is the multiplier; the first six products of the sequence are: $10,30,90,270,810,2430$.

Another solved case of a periodic sequence is that of $1 / 358=0.0028011204480 \ldots$ The specifications are: box- 4 , one-digit dendrite (0), prime number 7 the base, product seed is $280\left(2^{3} \times 5 \times 7\right)$, and 4 is the multiplier. The three first numbers accumulating are: $280,1120=280 \times 4$, and $4480=1120 \times 4$.

Another case solved is the inverse of $989 ; 1 / 989=$ $0.00101112234580384 \ldots$ a greater than 75 period sequence. The first three boxes are filled with numbers in sequence $(10,11,12)$, whereas the rest of the boxes (at least up to the next eight) are filled with products of the number $23(2 \times 12-1)$. The sequence of products is: $23,45,91,184,368, \ldots$ The specifications of the sequence are: box-2, eight-digit dendrite (itself a sequence, as indicated), prime number 23 is the base, also being the product seed, number 2 is the multiplier. There could be more breaks in this sequence, which the author (due to the computing limitations used) could not detect.

$1 / 35=0.0285714285714 \ldots$ is a $6-n$ cycle, which involves accumulations of products of 28 ; the exact specifications are: box-2, one digit dendrite $(0)$, prime number 7 is the base $\left(35=5 \times 3^{2}\right)$, product seed is $28\left(7 \times 2^{2}\right)$, and 2 is the multiplier. The first five numbers accumulating are: $28,56,112,224$ and 448 .

The 6- $n$ period sequence of the inverse of 28 is $1 / 28=0.0357142857142857 \ldots$; this is an accumulation of products of 14 . The exact specifications are: box-2, four-digit dendrite $(0357=51 \times 7)$, prime number 7 is the base, 14 is the product seed, number 2 is the multiplier; the first five numbers accumulating are: 14, 28, 56, 112 and 224.

Another type of accumulation is that of the periodic sequence $1 / 38=0.02631578947 \ldots$ Here, the sequence is: $26,2 \times 26-5^{0}=31,2 \times 31-5^{1}=$ $57,2 \times 57-5^{2}=89,2 \times 89-5^{3}=53,2 \times 53-5^{4}=$ $-519,-2 \times 519+5^{4}, \ldots$ (yet to be studied). Also, sequences of some interest along similar lines are those involving the inverse of $38=2 \times 19$, and $26=$ $2 \times 13$. They are left to the interested reader to unfold, as an exercise.

\section{CONCLUSIONS: THE IMPOSSIBILITY OF CHAOS IN SOCIO-SPATIAL DYNAMICS}

The paper presented a conjecture, namely that if rational numbers are any indication, then one ought not to expect mathematical chaos in sociospatial (and even possibly, natural science) systems. In particular, impossibility of chaotic dynamics in socio-spatial systems at least is partly supported 
by the empirical fact that smooth variations in any social parameter does not generate period doubling behavior. No empirical evidence, to this author knowledge, has been presented to violate this assertion.

A second finding of possible interest has been that of "cycle accumulation" found in seemingly random sequences of periodic motions in the decimals of rational numbers. This finding might suggest that socio-spatial (and possibly natural science) systems are governed by the presence of simultaneous cycles ranging from temporal ones (hourly, daily, weekly, monthly, yearly, or seasonal cycles), as well as spatial ones (urban, regional, national, global cycles).

Obviously, the next step in this line of inquiry would be to consider the role of nonrational numbers and their relationship to rational ones in reference to the chaotic sequence of decimals they possess (such as the sequence of decimals in $\pi$ ). A second extension of this work might also be the use of these findings to better understand the role of approximations; necessary approximations found in the recordings of rational numbers might prove that all socio-spatial systems are properly to be characterized as "simulated or modeled" rather than "real." In effect, this approximation might rob all socio-spatial systems from their "reality," no matter the tool used to study and analyze them.

\section{Acknowledgments}

This work was inspired by and is dedicated to my daughter Daphne Iris.

The author wishes to thank his Research Assistant Chaoche (Kevin) Hsu for the computer work in generating the 75 digit decimal streams. Useful suggestions from Michael Sonis are also greatly acknowledged. The usual caveats apply.

\section{References}

Adams, W.W. and Goldstein, L.J. (1976). Introduction to Number Theory, Prentice-Hall, Englewood Cliffs, New Jersey.

Hao, B.L. (1983). Chaos, World Scientific, Singapore.

Metropolis, M., Stein, M.L. and Stein, P.R. (1973). On finite limit sets for transformations on the unit interval, Journal of Combinatorial Theory, 15, 25-44.

Morse, M. (1921). Recurrent geodesics on a surface of negative curvature, Trans. Am. Math. Soc., 22, 84-100.

Niven, I. (1961). Numbers: Rational and Irrational, Random House, New York.

Schroeder, M. (1991). Fractals, Chaos, Power Laws, W.H. Freeman and Co., New York.

Thue, A. (1906). Uber die gegenseitige lage gleicher Teile gewisser Zeichenreichen, K. Nord. Vid. Skrifter I Nat (Oslo), $7,1-22$.

Ulam, S. (1964). Computers, Special Issue on Mathematics and the Modern World, Scientific American, September, 181-198. 


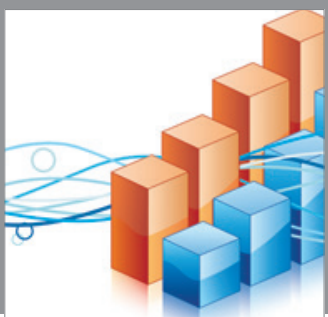

Advances in

Operations Research

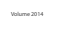

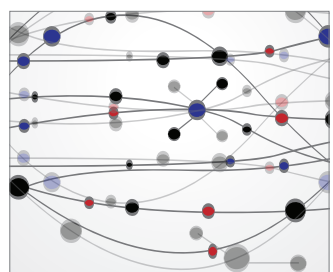

\section{The Scientific} World Journal
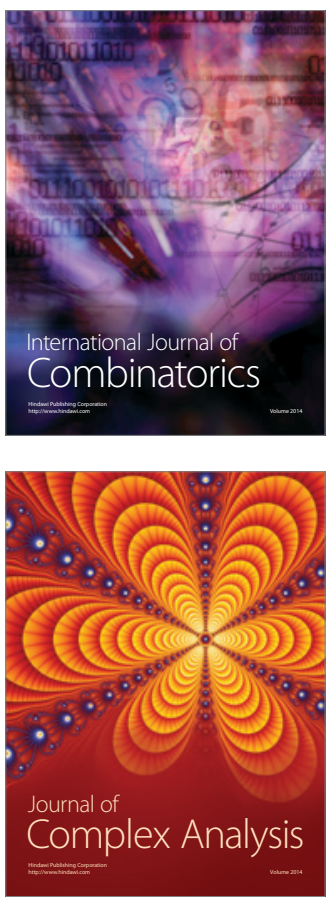

International Journal of

Mathematics and

Mathematical

Sciences
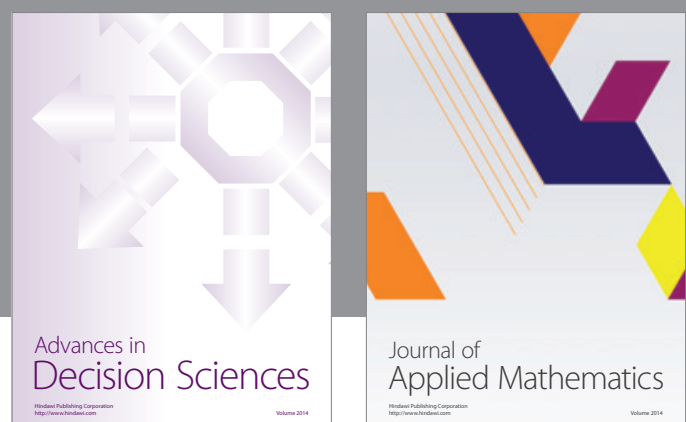

Journal of

Applied Mathematics
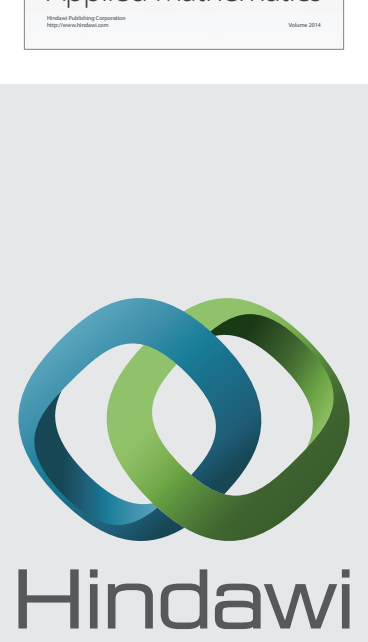

Submit your manuscripts at http://www.hindawi.com
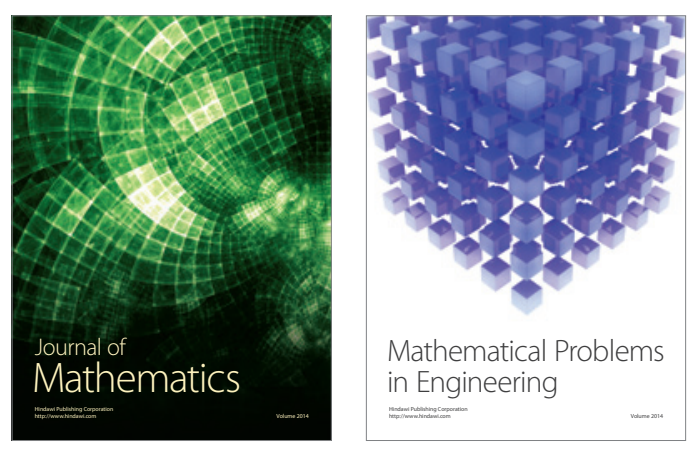

Mathematical Problems in Engineering
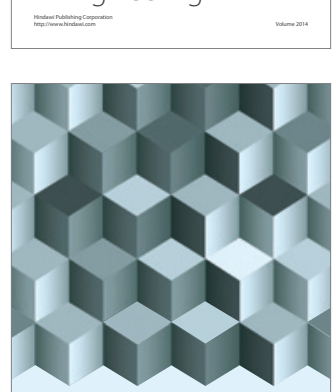

Journal of

Function Spaces
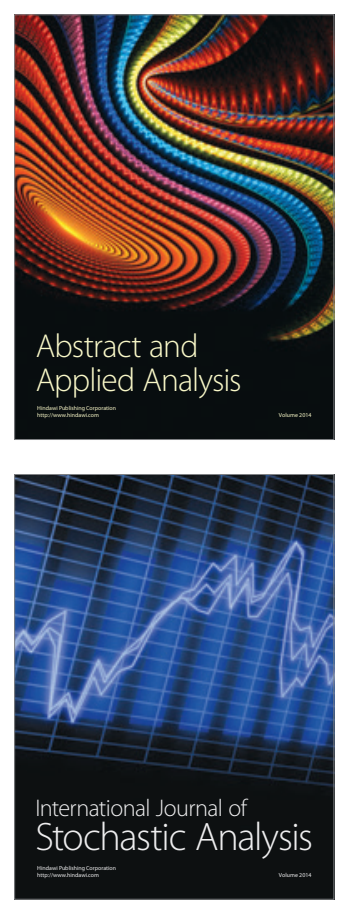

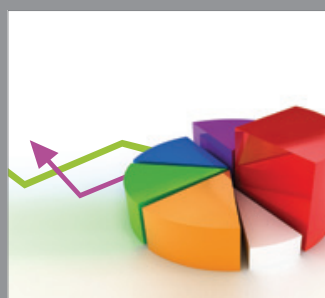

ournal of

Probability and Statistics

Promensencen
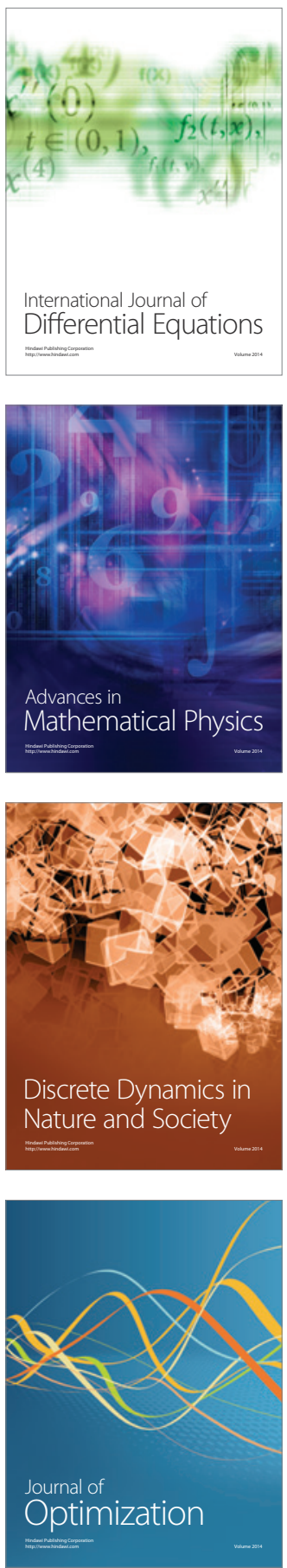